\title{
Estudo retrospectivo da morbidade e mortalidade associada com anestesia geral inalatória em cães
}

\section{Retrospective study of the mortality and morbidity associated with general inhalant anesthesia in dogs}

\author{
${\text { Roberta } \text { Carareto }^{1 *} \text {; Lígia de Souza Rocha }}^{2}$; Piedad Natália Henao Guerrero ${ }^{3}$; Marlos \\ Gonçalves Sousa ${ }^{4}$; Newton Nunes ${ }^{5}$; Danielli Parrilha de Paula ${ }^{6}$; Celina Tie Nishimori ${ }^{6}$
}

\section{Resumo}

\begin{abstract}
Foram avaliados os prontuários de 1.153 cães submetidos a anestesia geral inalatória no Hospital Veterinário Governador Laudo Natel, da Universidade Estadual Paulista, Campus de Jaboticabal-SP, no período de Julho de 2000 a Janeiro de 2003. Objetivou-se determinar a incidência de mortalidade e morbidade, tentando correlacioná-las com os agentes anestésicos empregados para manutenção anestésica e a classificação do paciente de acordo com a American Society of Anesthesiologists (ASA). A taxa de morbidade e mortalidade foi de, respectivamente, $9,19 \%$ e $1,13 \%$. A análise dos dados revelou que as complicações e óbitos podem ocorrer em pacientes cuja previsão de risco é inicialmente pequena, ou ainda naqueles onde os agentes anestésicos considerados mais seguros e estáveis foram utilizados. Entretanto, de acordo com a análise estatística, não houve correlação entre morbidade ou mortalidade e a classificação ASA, do paciente ou o agente anestésico utilizado.

Palavras-chave: Complicações anestésicas, taxa de mortalidade, anestesia geral inalatória
\end{abstract}

\begin{abstract}
The records of 1153 dogs that underwent inhalant general anesthesia at the Veterinary Teaching Hospital of São Paulo State University (UNESP, Campus of Jaboticabal, São Paulo, Brazil) from July 2000 until January 2003 were reviewed. We aimed at determining the mortality and morbidity rates, as well as their correlation with inhalation agents used for anesthetic maintenance, and the American Society of Anesthesiologists (ASA) classification of the patients. The overall morbidity rate was $9.19 \%$, whereas mortality rate was $1.13 \%$. Although the data analysis showed the occurrence of complications and death in patients initially thought to be risk free and in patients where safe anesthetic agents were used, no statistical correlation was determined to exist between morbidity or mortality and either the ASA classification of the animals or the anesthetic agent.

Key words: Anesthetic complications, mortality rate, inhalational general anesthesia
\end{abstract}

\footnotetext{
1 Doutoranda em Cirurgia Veterinária - Faculdade de Ciências Agrárias e Veterinárias (FCAV) - Universidade Estadual Paulista (UNESP) - Campus de Jaboticabal - SP E-mail: robertacarareto@hotmail.com

2 Autônoma, Ribeirão Preto - SP

3 Residente em Anestesiologia Veterinária, Kansas State University - Manhattan, KS, EUA

${ }^{4}$ Doutorando em Medicina Veterinária (Clínica Médica Veterinária) - FCAV - UNESP - Campus de Jaboticabal - SP

5 Professor Assistente - Departamento de Clínica e Cirurgia Veterinária - FCAV - UNESP - Campus de Jaboticabal - SP

6 Doutorandas em Cirurgia Veterinária - FCAV - UNESP - Campus de Jaboticabal - SP

* Autor para correspondência.
} 


\section{Introdução}

Os avanços na Medicina Veterinária e o maior cuidado dispensado pelos proprietários aos seus animais têm resultado em um incremento na expectativa de vida de cães e gatos nos últimos tempos. Dessa forma, animais de estimação têm apresentado maior probabilidade de, em algum momento de suas vidas, serem submetidos a algum tipo de intervenção cirúrgica e anestésica, as quais, por mais seguras que possam ser, sempre oferecem algum grau de risco ao paciente. Entretanto, a partir do momento em que temos consciência de quais são as principais falhas e, em quais situações elas costumam acontecer dentro de um serviço de anestesiologia, podemos minimizar os risco inerentes aos procedimentos e oferecer mais segurança aos nossos pacientes.

Com esse trabalho objetivou-se, por meio do estudo retrospectivo de prontuários anestésicos, determinar a incidência de mortalidade e morbidade em procedimentos cirúrgicos, a fim de elaborar uma análise crítica da qualidade do serviço de Anestesiologia Veterinária. Procurou-se ainda, correlacionar os agentes anestésicos voláteis utilizados e a classificação do paciente de acordo com a American Society of Anesthesiologists (ASA), com a taxa de óbito e complicações, a fim de tentar esclarecer quando as principais falhas ocorrem.

\section{Material e Métodos}

O estudo foi realizado de forma retrospectiva, avaliando-se os prontuários dos cães submetidos à anestesia geral inalatória no Hospital Veterinário Governador Laudo Natel, da Universidade Estadual Paulista (UNESP), Campus de Jaboticabal-SP, no período de Julho de 2000 a Janeiro de 2003. No referido período, foram anestesiados 1.153 cães, para realização de procedimentos cirúrgicos não-eletivos, assim sendo, não foram incluídos animais cujas cirurgias foram realizadas por conveniência.
De acordo com os prontuários, os animais foram classificados em uma escala de padrão de saúde, baseada nas categorias de risco estabelecidas pela American Society of Anesthesiologists (ASA), variando de ASA I a ASA V, sendo o paciente ASA I aquele que está aparentemente hígido, ao passo que o paciente ASA V apresenta-se moribundo e com expectativa de vida nula independentemente do procedimento cirúrgico a ser realizado.

Uma vez estabelecida a classificação ASA do paciente, analisou-se a incidência de complicações e a taxa de mortalidade dentro de cada categoria de risco. As complicações foram divididas em: arritmias cardíacas, apnéia, cianose, hipercapnia, hipotensão, instabilidade anestésica, parada cardiorrespiratória revertida e taquipnéia; as arritmias cardíacas foram subdivididas em: ausência de onda $\mathrm{P}$, bloqueio átrioventricular, bloqueio de ramo direito, bradicardia sinusal, extra-sístole ventricular, fibrilação ventricular, supradesnivelamento do segmento ST e taquicardia sinusal. Os pacientes foram ainda divididos em três grupos de acordo com o tipo de agente anestésico utilizado para manutenção do procedimento anestésico, ou seja Halotano, Isofluorano e Sevofluorano; para que fosse possível analisar os índices de mortalidade e morbidade ocorridos com cada agente.

Para análise estatística dos dados, empregou-se o coeficiente de correlação de Pearson, sendo as diferenças consideradas significativas quando $\mathrm{P}<0,05$.

\section{Resultados e Discussão}

Dentre os animais incluídos no estudo, 352 foram classificados como ASA I, 356 como ASA II, 316 como ASA III, 120 como ASA IV, e 9 como ASA V.

Quanto ao agente utilizado, 257 cães foram anestesiados com halotano; 421 com isofluorano; e 475 com sevofluorano.

As complicações ocorreram em 106 cães $(9,19 \%)$, sendo que nestes, o halotano foi empregado 
em 27 animais (25,47\%); isofluorano em 23 animais (21,70\%); e sevofluorano em 56 animais $(52,83 \%)$ (Tabela 1). Ao analisar estatisticamente os dados, não se evidenciou correlação $(\mathrm{P}=0,5846)$ entre a ocorrência de complicações e o agente anestésico utilizado. Além disso, as complicações foram verificadas em $18,87 \%, 26,42 \%, 37,73 \%, 16,03 \%$ e 0,95\% dos pacientes ASA I, II, III, IV e V, respectivamente (Tabela 2). Estes dados também foram submetidos à análise estatística, obtendo-se $\mathrm{P}=0,1019$, o que também não indicou correlação entre a ocorrência de complicações e a classificação do paciente de acordo com a ASA.

Tabela 1. Complicações ocorridas durante procedimentos anestésicos em cães, relacionadas ao agente anestésico volátil utilizado.

\begin{tabular}{lccc}
\hline \multicolumn{1}{c}{ Complicações } & Halotano & Isofluorano & Sevofluorano \\
\hline Apnéia & 1 & 1 & 2 \\
Arritmias cardíacas & & 1 & \\
$\quad$ Ausência de onda P & 3 & 4 & 7 \\
$\quad$ Bloqueio átrio-ventricular & 1 & & 18 \\
$\quad$ Bloqueio de ramo direito & 10 & 4 & 15 \\
$\quad$ Bradicardia sinusal & 3 & & 1 \\
$\quad$ Extra-sístole ventricular & & 1 & 4 \\
$\quad$ Fibrilação ventricular & 3 & 1 & 3 \\
$\quad$ Supradesnivelamento ST & 1 & 2 & 4 \\
$\quad$ Taquicardia sinusal & 1 & 1 & 1 \\
Cianose & 2 & 2 & 56 \\
Hipercapnia & 1 & & 4 \\
Hipotensão & 1 & 23 & \\
Instabilidade anestésica & 27 & & \\
Parada cardiorrespiratória revertida & & & \\
Taquipnéia & & & \\
Total & & &
\end{tabular}

Tabela 2. Complicações ocorridas durante procedimentos anestésicos em cães, relacionadas à classificação da ASA do paciente.

\begin{tabular}{|c|c|c|c|c|c|}
\hline Complicações & ASA I & ASA II & ASA III & ASA IV & ASA V \\
\hline Apnéia & 1 & & 2 & 1 & \\
\hline \multicolumn{6}{|l|}{ Arritmias cardíacas } \\
\hline Ausência de onda P & & & 1 & & \\
\hline Bloqueio átrio-ventricular & 2 & 7 & 5 & & \\
\hline Bloqueio de ramo direito & 1 & & & & \\
\hline Bradicardia sinusal & 8 & 12 & 13 & 1 & \\
\hline Extra-sístole ventricular & 2 & 4 & 9 & 6 & 1 \\
\hline Fibrilação ventricular & & & & 1 & \\
\hline Supradesnivelamento ST & & & 1 & & \\
\hline Taquicardia sinusal & 4 & & & & \\
\hline Cianose & & 2 & 1 & 3 & \\
\hline Hipercapnia & 1 & & 3 & 2 & \\
\hline Hipotensão & & & 3 & & \\
\hline Instabilidade anestésica & 1 & 2 & 2 & & \\
\hline Parada cardiorrespiratória revertida & & & & 2 & \\
\hline Taquipnéia & & 1 & & 1 & \\
\hline Total & 20 & 28 & 40 & 17 & 1 \\
\hline
\end{tabular}


Mortalidade foi observada em 13 animais (1,13\%), tendo sido utilizado o halotano em 3 cães $(23,08 \%)$, o isofluorano em 5 (38,46\%), e o sevofluorano em 5 $(38,46 \%)$. O tratamento estatístico revelou $\mathrm{P}=0,1529$, indicando que a mortalidade não está correlacionada com o agente utilizado. Dentre estes animais, 5 eram ASA II; 5 eram ASA III e 3 eram ASA IV. Novamente, não houve correlação $(\mathrm{P}=0,4391)$ entre a ocorrência de óbitos e a classificação ASA do animal. Todos os pacientes ASA V deste estudo foram eutanasiados durante o período perioperatório devido à constatação de enfermidade grave, não passível de correção, e desta forma não foram inclusos na contagem da mortalidade.

Comparar as complicações entre estudos retrospectivos realizados é relativamente difícil, visto que nem todos os trabalhos levam em consideração os mesmos parâmetros. Nosso índice de 9,19\% de complicações observadas em cães confronta com um estudo feito por Gaynor et al. (1999), que registraram $12,00 \%$ de morbidade.

Ainda que a correlação entre o agente anestésico utilizado para manutenção e a taxa de complicação não foi significativa, observou-se que a maioria das complicações ocorreu com o sevofluorano. Talvez, este surpreendente fato possa ter sido causado porque a maioria dos pacientes classificados como ASA IV (isto é, animais clinicamente instáveis com doenças sistêmicas graves) foi anestesiada com este agente. Por outro lado, o halotano, que mais acentuadamente sensibiliza o miocárdio à ação das catecolaminas e causa maior depressão cardiorrespiratória (MORGAN; MIKHAIL, 1996), associou-se a um menor índice de morbidade. Este resultado também pode ser compreendido ao se observar que a maioria dos pacientes classificados como ASA I foi anestesiada com tal agente.

O tempo de cirurgia também foi levado em conta para se proceder a escolha do agente a ser utilizado para manutenção anestésica. Assim sendo, o isofluorano e o sevofluorano foram os halogenados de escolha em se tratando de cirurgias mais complicadas e longas. Isso pode ter contribuído para que as complicações fossem mais freqüentemente observadas com estes agentes. Analisando os fatores de risco perioperatório, Schwilk et al. (1990) verificaram que as complicações aumentam significativamente depois que o procedimento cirúrgico tem duração superior a 30 minutos.

Dentre os 21,7\% de complicações verificadas com o isofluorano, a maioria foi devido à depressão respiratória. Uma vez que a depressão respiratória está intimamente correlacionada com planos anestésicos profundos (HALL; CLARKE, 1983), este fato pode ser explicado por ser tratar de um agente que tem um coeficiente de solubilidade sangue/gás significativamente baixo (HALL; CLARKE; TRIM, 2001), sendo capaz de aprofundar rapidamente a anestesia. Em estudo feito por Dohoo (1990), foi verificado que o isofluorano associava-se com as complicações ocorridas numa freqüência 2,6 vezes mais do que o esperado.

A análise estatística também não revelou correlação significativa entre a ocorrência de complicações e a classificação ASA do paciente. Apesar da ausência de significância matemática, clinicamente observou-se maior morbidade entre os pacientes ASA II e ASA III. No caso específico dos pacientes ASA II, este valor não seria esperado, visto que são considerados como pacientes de menor risco (SCHNEIDER, 1983). Entretanto, apesar da classificação ASA servir de parâmetro para previsão do risco, este sistema é limitado devido à dificuldade de se categorizar o paciente (DYSON; GRANT MAXIE; SCHUNURR, 1998). Além disso, o mesmo autor comenta que o preparo pré-operatório também pode diminuir a categoria de risco do paciente. É válido lembrar que todos os pacientes submetidos à anestesia podem apresentar eventuais intercorrências, mesmo que sejam classificados como ASA I e II (FUTEMA, 2002).

A mortalidade observada de 1,13\% confronta com dois estudos semelhantes realizados na Universidade do Colorado, que demonstram queda da mortalidade 
de $1,2 \%$ na década de 50 , para $0,43 \%$ na década de 80 (LUMB; JONES, 1984 apud GAYNOR; DUNLOP; WAGNER, 1999). Estudo realizado no Canadá por Dyson, Grant Maxie e Schunurr (1998) revelou taxa de mortalidade de $0,11 \%$, mas $92 \%$ dos animais incluídos na análise foram classificados como ASA I ou II. Outro estudo realizado por Fantoni e Futema (1997) determinou uma taxa de mortalidade de $0,75 \%$. Neste trabalho, a taxa de mortalidade foi superior aos estudos reportados previamente. Talvez esse resultado possa ser explicado se levarmos em conta que nenhum paciente catalogado foi anestesiado para cirurgia eletiva ou de conveniência. Assim sendo, todos os pacientes eram portadores de algum tipo de afecção cirúrgica, que de alguma forma pode ter contribuído para alterar a homeostase do organismo e conseqüentemente, favorecer o aparecimento das complicações.

Apesar da inexistência de correlação entre a classificação ASA dos cães e a ocorrência de mortalidade, observou-se maior ocorrência entre os pacientes ASA II e III. De acordo com a classificação ASA, pacientes ASA IV e $\mathrm{V}$ apresentam maior risco de virem a óbito (VACANTI; VAN HOUTEN; HILL, 1970), mas eventuais complicações podem estar presentes nos animais anestesiados ainda que estejam classificados como ASA I ou ASA II (FANTONI, 1997). Em estudo similar, Fantoni (1997) demonstrou que $22,2 \%$ dos óbitos ocorreram em pacientes classificados como ASA II, III e IV e $33,3 \%$ em ASA V.

O fato da maior taxa de mortalidade ter ocorrido com o sevofluorano talvez possa ser explicado pela utilização deste agente na maioria dos pacientes que apresentavam doença sistêmica grave associado a um quadro clínico instável.

Paralelamente, é importante ressaltar, que os resultados deste estudo podem ter sofrido influência de uma série de variáveis que não foram analisadas, tais como: idade, raça, sexo, tempo cirúrgico, tipo de cirurgia, medicação pré-anestésica e agentes indutores. Pois como se trata de um estudo clínico, baseado na rotina de um hospital, a padronização dos grupos não pôde ser feita, a semelhança de estudos experimentais. Ainda assim tais resultados não devem ser desprezados, mas sim servir de base para que outros estudos retrospectivos sejam feitos periodicamente, pois é por meio destes trabalhos que conseguimos fazer uma análise da qualidade das anestesias. E ainda, são eles que, eventualmente, nos esclarecem como e quando as falhas acontecem, para que num segundo momento seja possível preveni-las.

\section{Conclusões}

De acordo com os dados analisados, é possível concluir que complicações e óbitos podem ocorrer em pacientes cuja previsão de risco, determinada pela classificação ASA, é inicialmente pequena. Também mostra que por mais seguros e estáveis que possam ser os agentes anestésicos, necessita-se de muito critério e cautela durante a utilização dos mesmos. Entretanto, de acordo com a análise estatística, não houve correlação entre morbidade ou mortalidade e a classificação ASA, do paciente ou o agente anestésico utilizado.

\section{Referências}

DOHOO, S. E. Isoflurane as an inhalational anesthetic agent in clinical practice. Canadian Veterinary Journal, Ottawa, v.31, p.847-850, 1990.

DYSON, D. H.; GRANT MAXIE, M.; SCHNURR, D. Morbidity and mortality associated with anesthetic management in small animal veterinary practice in Ontario. Journal of the American Animal Hospital Association, Lakewood, v.34, n.4, p.325-335, jul./aug. 1998.

FANTONI, D. T.; FUTEMA, F. Estudo das causas de óbito perioperatório no ato anestésico-cirúrgico de pequenos animais no HOVET-USP. In: ENCONTRO DE ANESTESIOLOGIA VETERINÁRIA, 3., 1997, Araçatuba. Anais...Araçatuba: Unesp, 1997.

FUTEMA, F. Avaliação pré-anestésica. In: FANTONI, D. T.; CORTOPASSI, S. R. G. Anestesia em cães e gatos. São Paulo: Roca, 2002. p.59-63. 
GAYNOR, J. S.; DUNLOP, C. I; WAGNER, A. E.; WERTZ, E. M; GOLDEN, A. E.; DEMME, W. C.

Complications and mortality associated with anestesia in dogs and cats. Journal of the American Animal Hospital Association, Lakewood, v.35, 1999. p.13-17.

HALL, L. W.; CLARKE, K.W. Accidents and emergences associated with anaesthesia. In: Veterinary anaesthesia. 8. ed. London: Baillière Tindall, 1983. p.367388.

HALL, L. W.; CLARKE, K. W.; TRIM, C. M. General pharmacology of the inhalation anaesthetics. In:

Veterinary Anaesthesia. 10 ed. London: W. B. Saunders, 2001.p. 133-147.

LUMB, W. V.; JONES, E. W. Statistics and records. In: . Veterinary anesthesia. 2. ed. Philadelphia: Lea

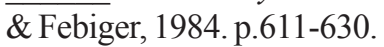

MORGAN, G. E.; MIKHAIL, M. S. Inhalational anesthetics. In: . Clinical Anesthesiology. 2. ed., Stamford: Appleton \& Lange, 1996. p.109-127.

SCHWILK, B.; FRIESS, L.; FRIESDORF, W; AHNEFELD, F. W; GEORGIEFF, M. Preoperative risk factors and intraoperative and postoperative risk management in 11.890 anesthesias. Initial results of a prospective study. Anasthesiol Intensivmed Notfallmed Schmerzther, Stuttgart,v. 28, n. 8, p.484-492, 1993.

SCHNEIDER, A. J. L. Assessment of risk factors and surgical outcome. Surgical Clinics of North America, Philadelphia,v.63, n.5, p.1113-1126, 1983.

VACANTI, C. J.; VAN HOUTEN, R. J.; HILL, R. C. A statistical analysis of the relationship of status to postoperative mortality in 68, 388 cases. Anesthesia Analgesia, Baltimore, v. 49, n. 4, p.564, 1970. 